\title{
Acupuncture on the Endometrial Morphology, the Serum Estradiol and Progesterone Levels, and the Expression of Endometrial Leukaemia-inhibitor Factor and Osteopontin in Rats
}

\author{
Houju Fu, ${ }^{1}$ Yuanqiao He, ${ }^{2}$ Ying Gao, ${ }^{1}$ Yicun Man, ${ }^{3}$ Wukun Liu, ${ }^{4}$ and Hua Hao \\ ${ }^{1}$ Department of Obstetrics and Gynecology, Union Hospital, Tongji Medical College, Huazhong University of Science and Technology, \\ Wuhan Hubei 430022, China \\ ${ }^{2}$ Department of Urology, Union Hospital, Tongji Medical College, Huazhong University of Science and Technology, \\ Wuhan Hubei 430022, China \\ ${ }^{3}$ Department of Gynecology, Endometriosis Research Center, Charité, Campus Benjamin Franklin, Hindenburgdamm 30, \\ 12200 Berlin, Germany \\ ${ }^{4}$ Institute of Pharmacy, Free University of Berlin, Koenigin Luise Strasse 2+4, 14195 Berlin, Germany \\ ${ }^{5}$ Department of Pathophysiology, Tongji Medical College, Huazhong University of Science and Technology, \\ Wuhan Hubei 430022, China
}

Correspondence should be addressed to Ying Gao, gaoyingxh@yahoo.com.cn

Received 2 February 2010; Revised 15 April 2010; Accepted 30 June 2010

Copyright (C 2011 Houju Fu et al. This is an open access article distributed under the Creative Commons Attribution License, which permits unrestricted use, distribution, and reproduction in any medium, provided the original work is properly cited.

\begin{abstract}
Although it is well known that acupuncture has beneficial effects on a variety of medical conditions especially in pain relief, nausea, and vomiting, it remains controversial whether it has positive impact on the female reproduction. The present study aimed to evaluate whether the following endometrial receptivity factors: the endometrial morphology, the hormone concentrations, and the protein expression of endometrial leukaemia-inhibitory factor (LIF) and osteopontin (OPN) could be improved by the acupuncture in clomiphene citrate(CC)-induced rat model during implantation period. Results showed that, compared with the CC group, glandular development advanced, the serum estradiol levels decreased significantly, and the glandular area and endometrial LIF and OPN expression were significantly higher in acupuncture group. There were no significant differences in serum progesterone levels, endometrial thickness, and stromal area between groups. These results suggest that acupuncture can improve certain aspects of endometrial receptivity in CC-induced rat model during implantation period, which might result in endometrial state better to female reproduction.
\end{abstract}

\section{Introduction}

Acupuncture (AC), one of the key components of Traditional Chinese Medicine (TCM), is used at certain points on the body that relate to special pathways known as meridians to mobilize the Qi (Chee) stuck, based on the notion that Qi, the vital energy of body health, circulates and moves freely along meridians to maintain the body in a "balanced state' between "Yin" and "Yang" [1]. This therapy is a safe and effective holistic medicine backed by more than three thousand years of practice and research and has been gaining increasing popularity in the west world as complementary and alternative therapeutic intervention [2].

$\mathrm{AC}$ has been used for a variety of medical conditions in both adults $[2,3]$ and children [4]: pain relief, nausea and vomiting, drug addiction, stroke rehabilitation, depression, irritable bowel syndrome, and asthma, to name a few. A National Institutes of Health consensus development panel released a survey of $\mathrm{AC}$, indicating that it has beneficial effects for surgery and chemotherapy-related nausea and vomiting and pain [5]. 
Recently, AC has been administrated in assisted reproductive technology to enhance the success of in vitro fertilization (IVF) treatment, due to the relatively low success rate of IVF per cycle, as well as the high emotional and financial costs. Some investigations have already demonstrated that AC had positive effects in anovulatory subfertility $[6,7]$, male subfertility $[3,8]$, pain relief during oocyte retrieval $[2,9,10]$, IVF implantation rates $[11,12]$, and the quality of life of patients undergoing IVF [2]. Also, there are reviews suggesting that certain effects of AC are needed by uterus [13] and it improves pregnancy rates and live birth among women undergoing IVF [14]. However, the definitive role of AC in infertility is still unclear and controversial $[2,3]$.

AC has specific Qi-Xue mechanism, channels, De-qi therapy, and manipulation, which are completely different from the ordinary drug administration. Its mechanism remains a mystery under the condition of current scientific technologies. Even, its placebo assessment has become a subject of controversy [15-18]. More clinical trials and basal researches that are consistent with TCM principles are required to provide a greater understanding for the clinical applicability of AC.

Clomiphene citrate (CC), a nonsteroidal compound, is one of the remarkably common drugs used in infertile women especially those with polycystic ovary syndrome, and is thought to have oestrogenic and antioestrogenic properties. Its antiestrogenic effect in hypothalamic and pituitary is exactly what we need, through inhibiting estrogen receptors to result in a favorable alteration in the characteristics of pulsatile gonadotropin-releasing hormone secretion and then the initiation of ovulation [19], while its interacting with other tissues such as ovary and endometrium may lead to underlying pathophysiologic negative influence on reproductive tract [20-22], which induce discrepancy between ovulation rate (60\%-85\%) and conception rate (20\%) [19].

The aim of this study was to investigate whether AC administration would improve these side effects of CC on the endometrium histology, endometrium receptivity cytokine, and the serum estradiol(E2) and progesterone(P) in CCinduced rat model and to provide evidence for the use of $\mathrm{AC}$ in reproduction.

\section{Materials and Methods}

2.1. Animals. Sexually mature female Sprague-Dawley rats, weighing 200-230 g were caged in animal colony according to the institutional guidelines established by the Animal Care and Use Committee of Tongji Medical College, Huazhong University of Science and Technology. The rats were fed with a rodent diet produced by school animal center and water freely, under the controlled temperature $\left(25 \pm 0.5^{\circ} \mathrm{C}\right)$ and a 12-hour light-dark regimen (lights on from 7:00 AM to 7:00 PM). The rodent diet is mainly made of corn, flour, dehydrated soybean meal, extruded soybean meal, fish meal, wheat bran, and alfalfa, according to the national standards, which do not affect hormonal level. The rats were housed for at least one week of acclimatization period before the experiment.
2.2. Tissue Preparation. Estrus was identified by daily vaginal smear. Only rats that had exhibited estrus for more than two consecutive periods of regular 4-day cycles were recruited into the present study. Suitable rats were randomly allocated to three groups, each including 11 subjects.

Rats in CC group were administered with Clomifene Citrate Tablets (Chinese name: Fa Di Lan. Codal Synto Limited, Cyprus) $100 \mathrm{mg} / \mathrm{kg} /$ day dissolved in $2 \mathrm{~mL}$ of tap water via lavage at 9.00 AM-11.00 AM for 2 days, beginning from the 1 st day of diestrus stage of estrous cycle. On day 3, they were mated after being given the intraperitoneal injection of human chorionic gonadotrophin (Livzon Pharmaceutical Group Inc., Guangdong, China) at $0.1 \mathrm{IU} / \mathrm{g}$ bw at 5.00 p.m. If sperms were observed in the vaginal smear specimens on the 4th day morning, we regarded that the rats were pregnant, and defined day 4 as the 1st day of pregnancy. In AC group, besides the procedures mentioned above, conscious rats which were swung in a self-made linen sack and kept in standing (prone) position were administrated with AC for 25 minutes in the afternoon once a day for 6 days, from the same day of CC administration to the 3rd day of pregnancy. The normal control (NC) group was composed of untreated pregnant rats, which were naturally mated during their estrus phases.

The three groups of rats were anesthetized with $10 \%$ Chloral Hydrate at noon on the 5th day of pregnancy. Bloods were drawn through cardiac puncture for measurement of serum E2 and P, and the sera were separated and stored at $-20^{\circ} \mathrm{C}$ until later assay. Then rats were sacrificed by cervical dislocation and the whole uteri were collected promptly, with the excess fat and connective tissue trimmered off. Left uterine horns were transected and fixed in $10 \%$ formalin for immunohistochemical study while the right samples including both endometrium and myometrium were stored at $-80^{\circ} \mathrm{C}$ until the extraction of protein. The study design was shown in Table 1.

2.3. AC Treatment. The $16 \mathrm{~mm}$ long sterile disposable filiform needles (Shanghai Taicheng Technology Development Co., Ltd, Shanghai, China) were inserted to depths of $1-7 \mathrm{~mm}$, then lifted, and twirled by hand evenly every 5 minutes. The stimulated points were as follows: bilateral Sanyinjiao (SP6), bilateral Zusanli (ST36), bilateral Taichong (LR3), Guanyuan (CV4), and Zhongji (CV3). The depth and the location of the points were based on the concepts of TCM, the atlas of skeleton and acupoints of rat [23], and the anatomical location described in previous research [24]. Sanyinjiao (SP6) is situated approximately $10 \mathrm{~mm}$ directly above the tip of the medial malleolus, on the posterior border of the tibia. Zusanli (ST36) is located $4-5 \mathrm{~mm}$ lateral to the anterior tubercle of the tibia, posterolaterally to the distal end of the cranial tuberosity of the tibia, in the tibialis anterior muscle, innervated by the deep peroneal nerve. Taichong (LR3) is on the dorsum of the hind limb, in the depression distal to the junction of the first and second metatarsal bones. Zhongji (CV3) is located at the point of $4 / 5$ down the ventral midline connecting the umbilicus to the pubic tubercle. Guanyuan (CV4) is located at the point of 3/5 down the ventral midline connecting the umbilicus to the pubic tubercle. 
TABLE 1: The design of this study.

\begin{tabular}{|c|c|c|c|c|c|}
\hline Day/group & Group CC & Group AC & Group NC & Estrus cycle & $\begin{array}{l}\text { Duration of } \\
\text { pregnancy }\end{array}$ \\
\hline Day 1 & $\mathrm{CC}(100 \mathrm{mg} / \mathrm{kg})$ & CC $(100 \mathrm{mg} / \mathrm{kg})$ acupuncture & & Diestrus & \\
\hline Day 2 & $\mathrm{CC}(100 \mathrm{mg} / \mathrm{kg})$ & CC $(100 \mathrm{mg} / \mathrm{kg})$ acupuncture & & Diestrus proestrus & \\
\hline Day 3 & HCG (0.1 IU/g) mated & Acupuncture HCG (0.1 IU/g) mated & Mated & Proestrus estrus & \\
\hline Day 4 & Found sperm & Found sperm acupuncture & Found sperm & & The 1st day \\
\hline Day 5 & & Acupuncture & & & The 2nd day \\
\hline Day 6 & & Acupuncture & & & The 3rd day \\
\hline Day 7 & & & & & The 4th day \\
\hline Day 8 & Drew blood collected uterus & Drew blood collected uterus & Drew blood collected uterus & & The 5th day \\
\hline
\end{tabular}

CC: clomiphene citrate; AC: acupuncture; NC: normal control; HCG: human chorionic gonadotrophin.

2.4. Radioimmunoassay (RIA). Sera E2 and P were analyzed by RIA kits (S10940094, Beijing North Institute of Biological Technology, Beijing, China) in the Department of Nuclear Medicine of Tongji Hospital. All sera were assayed on the same day to avoid interassay variation. The samples were analyzed in serial dilutions optimized to linear part of the standard curve and corrected for nonspeci?c binding. The intra-assay coef?cient of variations of both $\mathrm{E} 2$ and $\mathrm{P}$ were less than $10 \%$. For E2, the detection limit was less than $2 \mathrm{pg} / \mathrm{mL}$. The rates of cross reactivity of E2 with estriol, $P$ and testosterone were $0.016 \%, 0.01 \%$ and $0.01 \%$, respectively. The detection limit of $P$ was $2 \mathrm{ng} / \mathrm{mL}$ and the reference intervals were $0.2-100 \mathrm{ng} / \mathrm{mL}$.

2.5. Hematoxylin and Eosin Staining. After fixed with $10 \%$ formalin for 24 hours and embedded in paraffin, the uteri were cut into a thick $5 \mu \mathrm{m}$ transverse sections and then mounted on slides. Parts of these tissue sections were stained with hematoxylin (Harris) and eosin according to the standard procedure. Morphological changes were observed under light microscope, and morphometric parameters were evaluated by high-resolution medical color image automatic analysis system (HMIAS)-2000 (Wuhan Champion Image Technology Co., Ltd, China).

2.6. Immunohistochemistry. Other parts of sections were also stained using standard procedures of immunohistochemistry staining. Deparaffinized in xylene and dehydrated in a series of ethanol solutions, they were then covered in $0.3 \%$ hydrogen peroxide for 20 minutes to block endogenous peroxidases. Antigen retrieval was performed by incubating the sections in $10 \mathrm{mmol} / \mathrm{L}$ citrate buffer solution $(\mathrm{pH} 6.0)$ at $100^{\circ} \mathrm{C}$ for 5 minutes and cooling naturally at room temperature. The sections were incubated with leukaemia inhibitory factor (LIF) purified goat polyclonal antibody (SC-1336, Santa Cruz, CA, USA) or osteopontin(OPN) mouse-anti-rat monoclonal antibody (SC-21742, Santa) diluted 1 : 100 overnight at $4^{\circ} \mathrm{C}$, respectively. After washing in PBS, the sections were incubated with biotinylated goat antimouse IgG or rabbit antigoat IgG, followed by SABC Solution(Boster Biological Technology, Ltd. Wuhan, China) for 30 minutes at room temperature and diaminobenzidine (DAB Kit, Boster Biological Technology, Ltd. Wuhan, China) for about $10 \mathrm{~min}$, during which the brown colour was controlled under light microscope. Staining intensity of tissue sections was also evaluated by HMIAS-2000. The deeper the color shows, the greater the value of optical density becomes.

2.7. Western Blot. Rat endometrium tissues were homogenated and lysed in extraction buffer $(50 \mathrm{mmol} / \mathrm{L}$ Tris$\mathrm{HCl}$ (pH 7.4), $150 \mathrm{mmol} / \mathrm{L} \quad \mathrm{NaCl}, 1 \%$ NP-40, $0.5 \%$ sodium deoxycholic acid, $0.1 \%$ SDS, $100 \mu \mathrm{g} / \mathrm{mL}$ PMSF, and $100 \mu \mathrm{g} / \mathrm{mL}$ leupeptin). The protein concentration was quantified using the BCA protein assay kit (Prod\#23227, Pierce, IL, USA). After denatured through incubating at aqua bulliens for $5 \mathrm{~min}$, the protein was electrophoresed with sodium dodecyl sulphate-polyacrylamide gel electrophoresis (SDS-PAGE) using 10\% polyacrylamide gels and transferred to nitrocellulose membranes (1620115, Bio-Rad, Hercules, CA, USA). The membranes were blocked with $5 \%$ fatfree powdered milk in TBS-T $(10 \mathrm{mmol} / \mathrm{L}$ Tris, $150 \mathrm{mmol} / \mathrm{L}$ $\mathrm{NaCl}$, and $0.05 \%$ Tween-20, $\mathrm{pH} 8.0$ ) at room temperature for 1 hour and incubated overnight in $1 \%$ TBS-T at $4^{\circ} \mathrm{C}$ with LIF purified goat polyclonal antibody (SC-1336, Santa) or OPN mouse-anti-rat monoclonal antibody (SC-21742, Santa) diluted $1: 1000$. Following three washes with TBS$\mathrm{T}$, blots were incubated with the appropriate secondary antibody IgG-horseradish peroxidase conjugate at dilution of $1: 2000$ at room temperature for 1 hour. After final washing with TBST, the membranes were detected by enhanced chemiluminescence (Prod\#34079, Pierce) and exposed to $\mathrm{X}$-ray films. Expression of target proteins was internally normalized to $\alpha$-Tubulin (SAB3500023, Sigma, USA).

2.8. Statistical Analysis. Data were analyzed by SPSS 12.0 statistical software (SPSS Inc., Chicago, USA). The KruskalWallis test was used to detect overall differences among three groups, followed by Mann-Whitney U-test for multiple comparisons. Results are expressed as mean \pm SD. $P$ value $<.05$ was considered significant in $2 \times 2$ comparisons. 
TABLE 2: Comparison of the morphometric characteristics of endometria in rats during implantation period.

\begin{tabular}{lcccr}
\hline Characteristics & Normal control & Acupuncture & Clomiphene citrate & $P$ value \\
& $n=11$ & $n=11$ & $422.90 \pm 17$ & $.00^{\mathrm{a}}$ \\
\hline Thickness $(\mu \mathrm{m})$ & $621.25 \pm 16$ & $441.31 \pm 24$ & $0.13 \pm 0.03$ & $.07^{\mathrm{b}}$ \\
Glandular area $\left(\mathrm{mm}^{2}\right)$ & $0.21 \pm 0.03$ & $0.17 \pm 0.03$ & $.00^{\mathrm{a}}$ & $.01^{\mathrm{b}}$ \\
Stromal area $\left(\mathrm{mm}^{2}\right)$ & $2.50 \pm 0.35$ & $1.80 \pm 0.36$ & $1.76 \pm 0.36$ & $.00^{\mathrm{a}}$ \\
\hline
\end{tabular}

${ }^{a}$ Normal control group versus clomiphene citrate group.

${ }^{\mathrm{b}}$ Acupuncture group versus clomiphene citrate group.

TABLE 3: Comparison of the serum oestradiol and progesterone concentrations during implantation period in rat model.

\begin{tabular}{lcccc}
\hline Level & Normal control & Acupuncture & Clomiphene citrate & $P$ value \\
& $n=11$ & $n=11$ & $n=11$ & \\
\hline Estradiol $(\mathrm{pg} / \mathrm{mL})$ & $1472.00 \pm 657$ & $1899.73 \pm 730$ & $2617.63 \pm 795$ & $.00^{\mathrm{a}}$ \\
Progesterone $(\mathrm{ng} / \mathrm{mL})$ & $2.03 \pm 0.45$ & $1.53 \pm 0.60$ & $1.47 \pm 0.96$ & $.03^{\mathrm{b}}$ \\
\hline
\end{tabular}

${ }^{\mathrm{a}}$ Normal control group versus clomiphene citrate group.

${ }^{\mathrm{b}}$ Acupuncture group versus clomiphene citrate group.

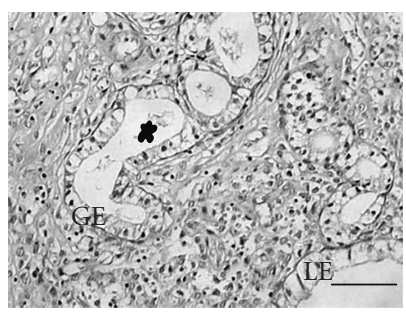

(a)

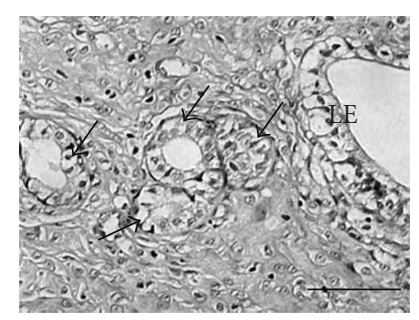

(b)

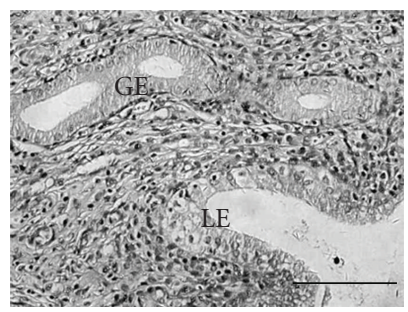

(c)

Figure 1: Comparison of endometrial morphology during implantation period in rats. Specimens from NC group (a) showed normal endometrium: well-arranged luminal epithelium (LE), abundant glandular secretions (asterisk in (a)) and coherent subnuclear vacuoles in both LE and glandular epithelium (GE). Endometrial tissues of intervention groups showed glandular-stromal dyssynchrony: no secretions but stroma similar to normal. Subnuclear vacuoles of CC group (c) were only shown in LE, but they appeared in both LE and GE after acupuncture treatment (arrows in (b)). Original magnification: $\times 200$. Scale bar $=100 \mu \mathrm{m}$.

\section{Results}

3.1. Endometrial Morphology. Only the endometrial layer was subjected to analysis, and the same outcome was obtained by two independent observers blind to treatment. Morphological changes were shown in Figure 1. Endometrial tissues from pregnant rats in NC group (Figure 1(a)) showed well-arranged luminal epithelium (LE), abundant glandular secretion and coherent subnuclear vacuoles in almost all LE and glandular epithelium (GE). There was no secretion in AC (Figure 1(b)) and CC groups (Figure 1(c)), and stromal morphology did not significantly differ among three groups. However, subnuclear vacuoles of AC group appeared in GE as well as in LE when compared with CC group.

Morphometric parameters and data of endometria detected were shown in Table 2. Glandular area differed significantly among the three groups (all $P<.05$ ). Endometrial thickness and stromal area were substantially different in the NC group than in the CC group (both $P<.01$ ), but not in the AC group than in the CC group (both $P>.05$ ).

3.2. E2 and P Concentrations in RIA. Table 3 summarized the serum hormone levels in the three groups during implantation period in rat model. The serum E2 concentration of the CC group was higher than that of the NC group $(P<.01)$, and the comparison of that between the AC and CC groups showed a significant decrease $(P<.05)$. However, the serum $P$ concentration did not significantly differ among the three groups in our present trial $(P>.05)$.

\subsection{Expression of Endometrial LIF and OPN Proteins in} Immunohistochemistry. The immunostaining for LIF (Figures 2(a)-2(d)) and OPN (Figures 2(e)-2(h)) was predominantly detected in GE and LE in rat endometrium, with a little expression in stroma. Staining intensity of LIF in AC group was weaker than that of NC group (optical density: $0.171 \pm 0.027$ versus $0.246 \pm 0.023)$, but higher than that of CC group (optical density: $0.135 \pm 0.023$ ). The same trend of immunoreactivity of OPN was observed in the three groups. (optical density: $0.287 \pm 0.022$ in NC group, $0.199 \pm 0.028$ in AC group, and $0.150 \pm 0.026$ in $\mathrm{CC}$ group). All the comparisons above had significant differences $(P<.01)$ and the staining intensities are depicted in Figure 3. 


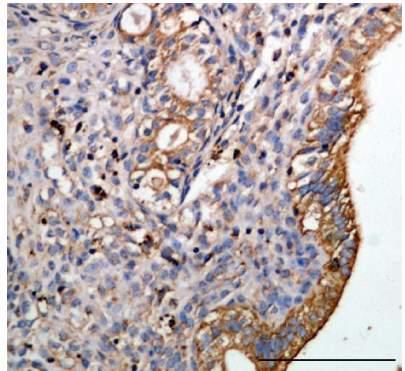

(a)

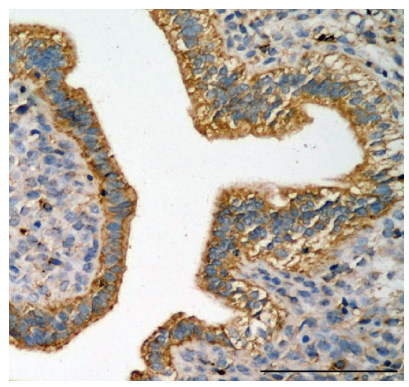

(e)

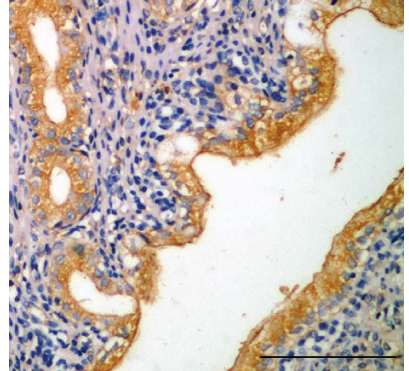

(b)

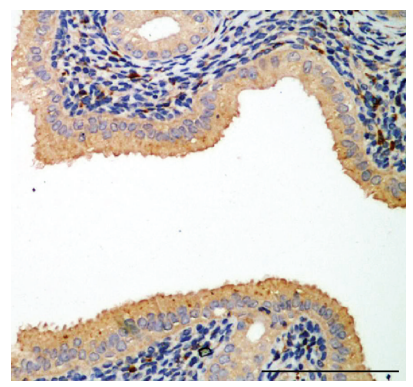

(f)

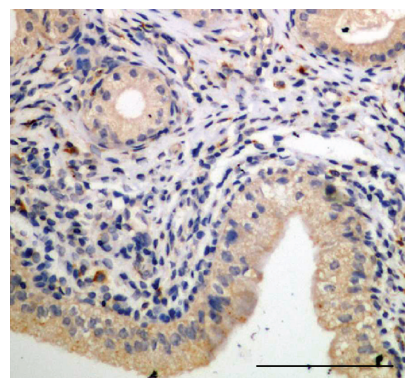

(c)

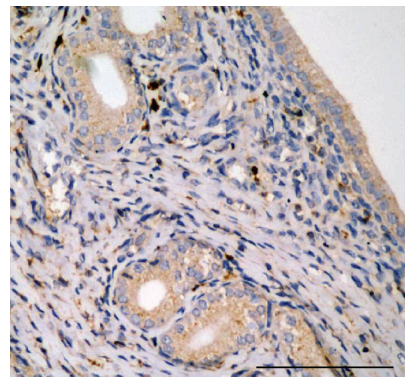

(g)

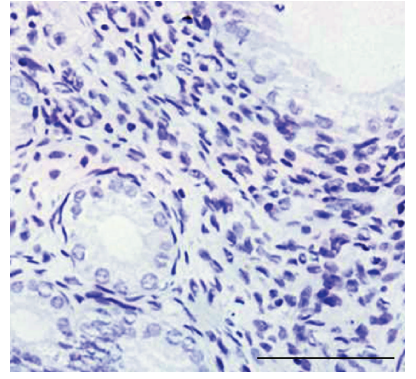

(d)

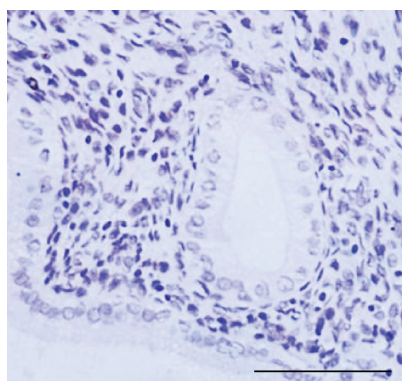

(h)

FIGURE 2: Immunohistochemical staining for the expression of endometrial leukaemia inhibitory factor (LIF) and osteopontin (OPN) during implantation period in rat. Note that immunostaining of the LIF $((a)-(d))$ and OPN $((e)-(h))$ was mainly detected on glandular and luminal epithelial cells. Staining intensity of both LIF and OPN in AC group was significantly weaker than that of NC group but stronger compared with CC group. When using PBS instead of the anti-LIF antibody (d) or the anti-OPN antibody (h), no positive signal was observed. ((a) and (e): NC group; (b) and (f): AC group; (c) and (g): CC group). Original magnification: $\times 200$. Scale bar $=100 \mu \mathrm{m}$.

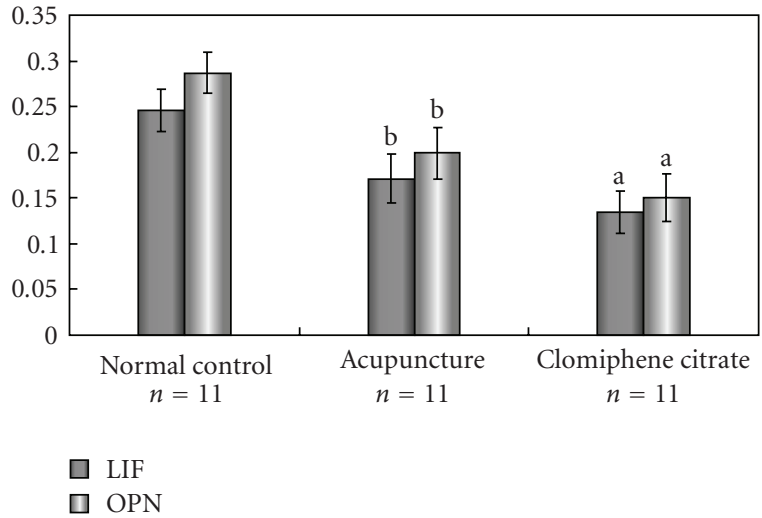

FIgURE 3: The immunohistochemical staining intensities of leukaemia-inhibitory factor (LIF) and Osteopontin (OPN) proteins in rat endometria during implantation period. Data are expressed as mean \pm SE. (a), $P<.01$ compared with Normal control group; (b), $P<.01$ compared with Clomiphene citrate group.

3.4. Expression of Endometrial LIF and OPN Proteins in Western Blot. Consistent with the results of immunohistochemical staining, the expression trends of LIF and OPN proteins were confirmed by Western blot analyses (Figure 4(a) and $4(\mathrm{~b}))$. The band of $\alpha$-Tubulin was used as an internal loading control in each lane. Normalized with $\alpha$-Tubulin expression level (Figures 4(c) and 4(d)), the expression of both LIF and OPN proteins in AC group were lower than those in the NC group, but higher as compared with CC group: $P$-values of $<.01$ in CC group versus NC group and $P$-values of $<.01$ in AC group versus CC group.

\section{Discussion}

Under the present high dose in our experiment, the rat model with high-serum E2, thin and impaired endometrium confirmed the side effects of CC [20-22] and resulted in abnormal endometrial receptivity which may cause the failure of approximately two-thirds of implantations [25]. The process of implantation, classically classified into three stages: apposition, adhesion, and invasion, involves a complex sequence of recognition signaling events between the synchronous stage conceptus and the primed and receptive uterine. It only takes place during the limited "implantation window", a restricted period of endometrial receptivity spanning, between days 20 and 24 of a regular menstrual cycle in humans $[26,27]$, days 4 and 6 of pregnancy in rats $[28,29]$. Endometrial receptivity that is rigorously controlled both temporally and spatially embraces various factors under the influence of ovarian hormones, including morphological features, cellular adhesion molecules family, cytokines, prostaglandins, and immunological regulation $[26,27]$, as is shown in Figure 5.

Endometrial morphology can be accomplished by histological dating of the endometrial biopsy specimen serving as an important bioassay. Not only there is evidence suggesting that sampling during the implantation window is quite 


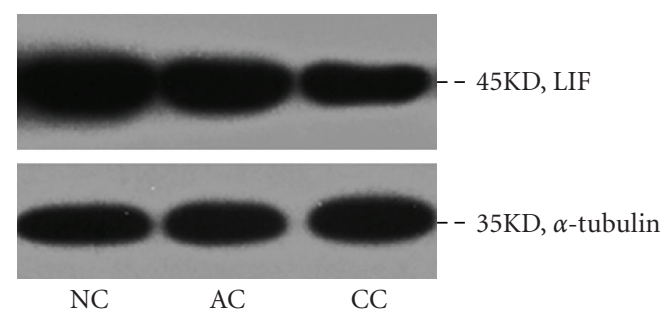

(a)

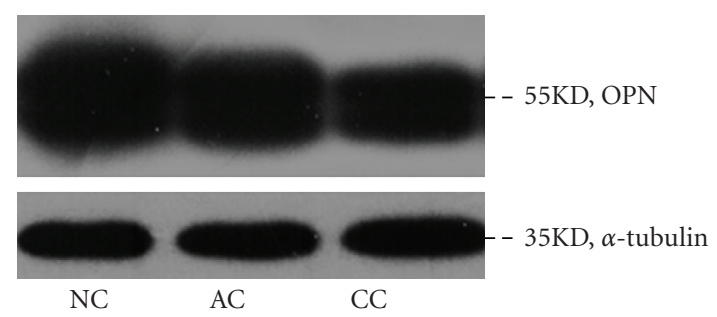

(b)

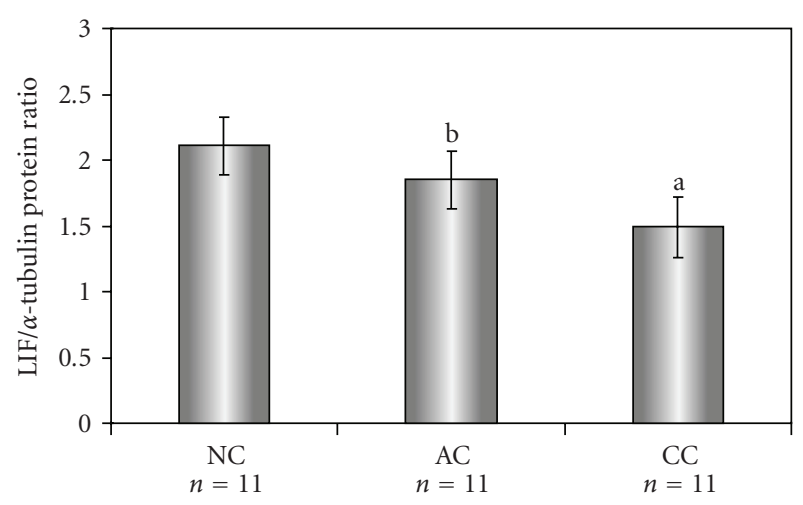

(c)

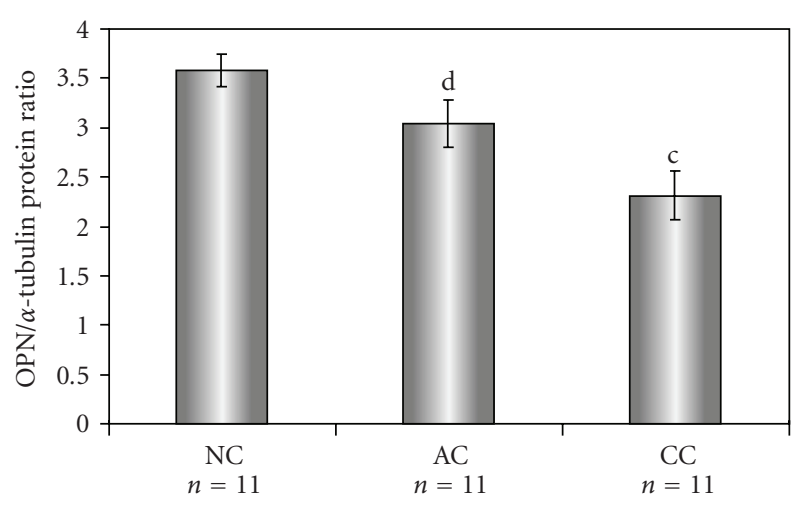

(d)

FIgURE 4: Expression of endometrial leukaemia-inhibitory factor (LIF) and osteopontin (OPN) proteins during implantation period in rats ((a) and (b)). Expression of target proteins was internally normalized to the optical density of $\alpha$-Tubulin ((c) and (d)). Data are expressed as mean \pm SE of the LIF/ $\alpha$-Tubulin protein ratio and OPN $/ \alpha$-Tubulin protein ratio: (a) and (c), $P<.01$ compared with NC group; (b) and (d), $P<.01$ compared with CC group. sensitive for identifying endometrial maturation [30], but some arguers, holding that the morphological change can't be used to assess the reproductive ability, also thought that the abnormal secretary endometrial maturation may adversely affect reproductive performance [31]. Previous researches found that $\mathrm{AC}$ administration had positive effect on morphology, such as the ultrastructural integrity of spermatozoa in human [32] and matured follicles rupture and conversion into corpus luteum in mice [33]. A pilot study has displayed that the combination of AC and Sildenafil suppositories increased endometrial thickness by upregulating nitric oxide synthase [34]. In terms of our data in present study, not the endometrial thickness $(P=.07)$ but the glandular area $(P=.01)$ of endometrium was significantly increased in the AC group compared with the CC group. So we suggested that AC may have capacity to mainly stimulate the growth of gland rather than stoma, which cannot be observed by ultrasound in clinics.

Sex steroids as systemic factors regulate the uterine receptivity and implantation process as well as menstruation through their ligand receptors expressed in the epithelial, stromal, and vascular cells. Estrogen enhances endometrial cell proliferation, and progesterone leads to the differentiation of these cells following ovulation. The latter hormone is required for implantation and pregnancy maintenance in all mammals, although the requirement for estrogen is species-specific [35]. In clinic, as we know, high E2 concentration will lead to ovary hyperstimulation syndrome. At present study, AC significantly suppressed the highserum E2 induced by CC to bring better implantation state. Preliminary studies have also found that AC was effective with the management of sex hormones. It could adjust serum follicle stimulating hormone (FSH), luteinizing hormone, and E2 in women suffering from ovulatory dysfunction [7], and in rats with primary dysmenorrheal [36]. Recently, in a randomized controlled trial, AC and auricular treatment significantly decreased the FSH level while increased the E2 level to relieve the menopausal hot flashes of the bilaterally ovariectomized women [37]. The serum concentrations of $\mathrm{P}$ in our study were similar among the three groups, which did not support the result described before [38], but we observed that the serum $P$ concentrations in six out of eleven samples of CC group were much lower than the normal level $(<1 \mathrm{ng} / \mathrm{mL})$, and the same phenomenon of CC-induced low $\mathrm{P}$ concentration has also been found in other studies [39, 40].

The present study showed that AC improved the expression of LIF and OPN proteins in a rat's uterus during implantation period. It must be closer to normal state. The two cellular factors largely accepted as the promising candidates of biomarkers of endometrium receptivity are critical to the establishment of blastocyst implantation and pregnancy. The important role for LIF was obviously shown on knockout mice's failure to implant, whereas LIF (-/-) blastocysts can be successfully transplanted into wild-type recipient females [41]. OPN acts as an adhesive, like a bridge between its ligand integrin $\alpha \mathrm{v} \beta 3$ on the embryo and epithelium [42]. OPN mRNA was highly expressed in human endometrium during periimplantation period or implantation window in several gene that profiling researches [43-45]. It has 


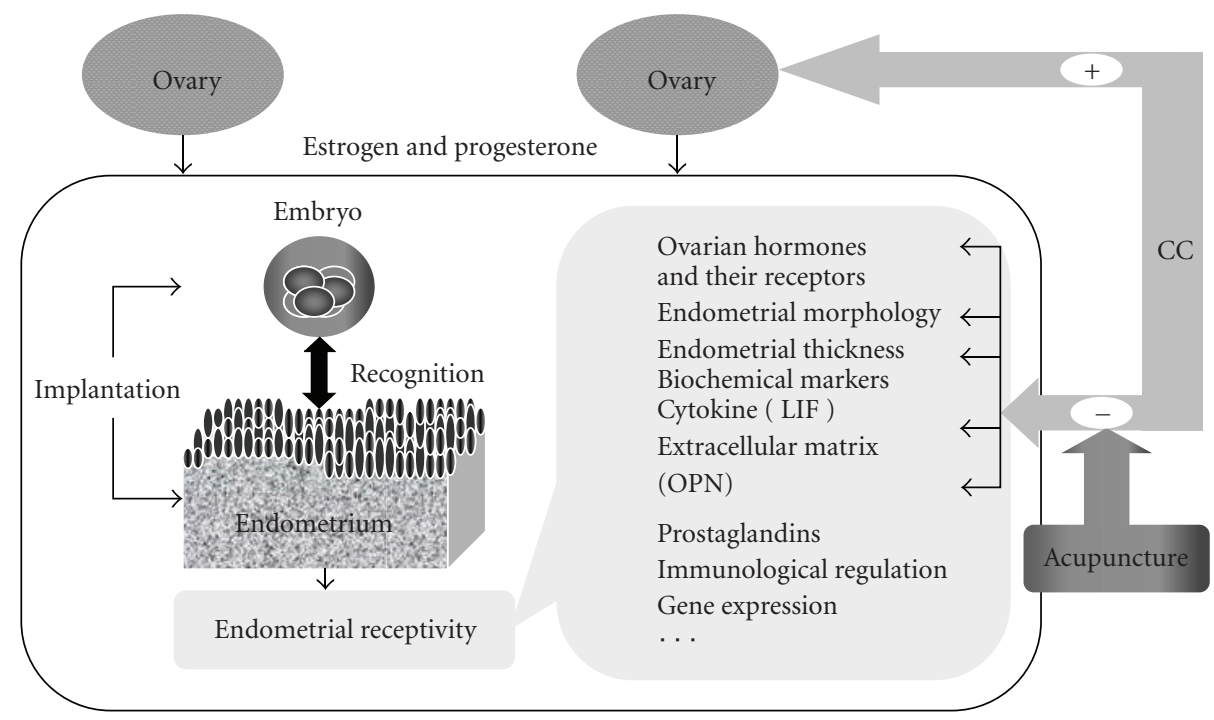

FIGURE 5: Under the influence of ovarian hormones, endometrial receptivity that is, rigorously controlled both temporally and spatially embraces various factors. Some of them, such as endometrial morphology, biochemical markers-leukaemia inhibitory factor (LIF), and osteopontin (OPN), as well as ovarian hormones are negatively influenced by clomiphene citrate (CC), which were improved by acupuncture in present trial. (+): The effect of CC on ovulation. (-): The adverse effects of CC on reproduction.

been demonstrated that AC stimulation affected cytokine production. Not only did it modulate immune Th1 and Th2 cytokines in the hypothalami of rats with lipopolysaccharideinduced fever [46] and in the ventral midbrains of healthy rats [47], but also increased the release of $\beta$-endorphin in the brain [48]. Besides, electro-AC has been found to raise the neuropeptide Y concentrations in follicular fluid [49] and reduce uterine motility in pregnant rats by inhibiting the cyclooxygenase- 2 expression of the endometrium [50].

The biological mechanisms of AC's impact on reproduction are still unclear. It has been established, however, that the positive effect of $\mathrm{AC}$ in the treatment of infertility is related to the following $[2,3]$ : (a) modulating the release of neurotransmitters, such as $\beta$-endorphin, which then influence the hypothalamic functions (b) increasing uterine and ovarian blood flow to improve endometrial environment (c) inhibiting uterine contraction to reduce the possibility of expelling embryo out of the uterus (d) modulating immune factors (e) reducing stress of subfertile patients. The acupoints chosen in the present trial have been found to be associated with regulating gonadal hormone levels in both human $[7,37]$ and animal $[36,51-63]$ and improving pregnancy rate of IVF $[11,12,14,54,55]$. According to the TCM principles, Sanyinjiao (SP6) is considered as a classic acupoint for female disorders and can soften and harmonize the liver and benefit the kidney Qi [56, 57]. Zusanli (ST36) is the main tonification acupoint for strengthening the body's resistance and restoring vital energy, and Taichong (LR3) spreads the stagnation of liver Qi and nourishes liver blood [57]. Both Zhongji (CV3) and Guanyuan (CV4) are on the Conception Vessel, which can nourish the uterus to adjust the axis function $[7,57]$. The days of AC administration were also in the light of previous studies in rat $[52,53]$. Recently, acupoint specificity [58-61] and sham needling [15-18] of
AC have been subjects of controversy, but there are studies that link particular acupoints with unique connective tissue location [62], the meridian with unique electrical property [63].In the experiment, we just use the normal pregnant rats as the control, because 'sham' designs study a simulacrum of AC, and a high-placebo model should be found [64].

In conclusion, $\mathrm{AC}$ was found in our study to ameliorate the uterine environment, by advancing the gland development, reducing the high-serum E2 concentration, and increasing the glandular area and the expression of receptivity markers: LIF and OPN proteins during the implantation period in CC-induced model. It may be a valuable complementary and alternative treatment for female reproduction. However, further rigorous research is needed to confirm this physiologic effectiveness of AC.

\section{Acknowledgments}

The authors are grateful to Dr. Jia Li (Hubei College of Traditional Chinese Medicine, Wuhan, China) and Dr. Fei Wang (Department of Acupuncture, Wuhan NO. One Hospital, Wuhan, China) for their guiding acupuncture technique. This paper was supported by Family Planning Science Foundation of Hubei Province (grant number: 4289).

\section{References}

[1] T. J. Kaptchuk, "Acupuncture: theory, efficacy, and practice," Annals of Internal Medicine, vol. 136, no. 5, pp. 374-383, 2002.

[2] B. J. Anderson, F. Haimovici, E. S. Ginsburg, D. J. Schust, and P. M. Wayne, "In vitro fertilization and acupuncture: clinical efficacy and mechanistic basis," Alternative Therapies in Health and Medicine, vol. 13, no. 3, pp. 38-48, 2007. 
[3] E. H. Y. Ng, W. S. So, J. Gao, Y. Y. Wong, and P. C. Ho, "The role of acupuncture in the management of subfertility," Fertility and Sterility, vol. 90, no. 1, pp. 1-13, 2008.

[4] J. I. Gold, C. D. Nicolaou, K. A. Belmont, A. R. Katz, D. M. Benaron, and W. Yu, "Pediatric acupuncture: a review of clinical research," Evidence-Based Complementary and Alternative Medicine, vol. 6, pp. 429-439, 2009.

[5] "Acupuncture: NIN consensus development panel on acupuncture," Journal of the American Medical Association, vol. 280, no. 17, pp. 1518-1524, 1998.

[6] E. Stener-Victorin, U. Waldenström, U. Tägnfors, T. Lundeberg, G. Lindstedt, and P. O. Janson, "Effects of electroacupuncture on anovulation in women with polycystic ovary syndrome," Acta Obstetricia et Gynecologica Scandinavica, vol. 79, no. 3, pp. 180-188, 2000.

[7] X. Mo, D. Li, Y. Pu, G. Xi, X. Le, and Z. Fu, "Clinical studies on the mechanism for acupuncture stimulation of ovulation," Journal of Traditional Chinese Medicine, vol. 13, no. 2, pp. 115119, 1993.

[8] J. Pei, E. Strehler, U. Noss et al., "Quantitative evaluation of spermatozoa ultrastructure after acupuncture treatment for idiopathic male infertility," Fertility and Sterility, vol. 84, no. 1, pp. 141-147, 2005.

[9] P. Humaidan and S. Stener-Victorin, "Pain relief during oocyte retrieval with a new short duration electro-acupuncture technique-an alternative to conventional analgesic method," Human Reproduction, vol. 19, no. 6, pp. 1367-1372, 2004.

[10] S. M. Sator-Katzenschlager, M. M. Wölfler, S. A. KozekLangenecker et al., "Auricular electro-acupuncture as an additional perioperative analgesic method during oocyte aspiration in IVF treatment," Human Reproduction, vol. 21, no. 8, pp. 2114-2120, 2006.

[11] W. E. Paulus, M. Zhang, E. Strehler, I. El-Danasouri, and K. Sterzik, "Influence of acupuncture on the pregnancy rate in patients who undergo assisted reproduction therapy," Fertility and Sterility, vol. 77, no. 4, pp. 721-724, 2002.

[12] S. Dieterle, G. Ying, W. Hatzmann, and A. Neuer, "Effect of acupuncture on the outcome of in vitro fertilization and intracytoplasmic sperm injection: a randomized, prospective, controlled clinical study," Fertility and Sterility, vol. 85, no. 5, pp. 1347-1351, 2006.

[13] R. Chang, P. H. Chung, and Z. Rosenwaks, "Role of acupuncture in the treatment of female infertility," Fertility and Sterility, vol. 78, no. 6, pp. 1149-1153, 2002.

[14] E. Manheimer, G. Zhang, L. Udoff et al., "Effects of acupuncture on rates of pregnancy and live birth among women undergoing in vitro fertilisation: systematic review and metaanalysis," British Medical Journal, vol. 336, no. 7643, pp. 545$549,2008$.

[15] I. Lund, J. Näslund, and T. Lundeberg, "Minimal acupuncture is not a valid placebo control in randomised controlled trials of acupuncture: a physiologist's perspective," Chinese Medicine, vol. 4, article 1, 2009.

[16] R. P. Dhond, N. Kettner, and V. Napadow, "Do the neural correlates of acupuncture and placebo effects differ?" Pain, vol. 128, no. 1-2, pp. 8-12, 2007.

[17] H. MacPherson, G. Green, A. Nevado et al., "Brain imaging of acupuncture: comparing superficial with deep needling," Neuroscience Letters, vol. 434, no. 1, pp. 144-149, 2008.

[18] T. Lundeberg, I. Lund, A. Sing, and J. Näslund, "Is placebo acupuncture what it is intended to be? "Evidence-Based Complementary and Alternative Medicine. In press.
[19] R. Homburg, "Clomiphene citrate-end of an era? A minireview," Human Reproduction, vol. 20, no. 8, pp. 2043-2051, 2005.

[20] E. Kousta, D. M. White, and S. Franks, "Modern use of clomiphene citrate in induction of ovulation," Human Reproduction Update, vol. 3, no. 4, pp. 359-365, 1997.

[21] S. Palomba, T. Russo, F. Orio Jr. et al., "Uterine effects of clomiphene citrate in women with polycystic ovary syndrome: a prospective controlled study," Human Reproduction, vol. 21, no. 11, pp. 2823-2829, 2006.

[22] A. J. Bonhoff, O. G. J. Naether, and E. Johannisson, "Effects of clomiphene citrate stimulation on endometrial structure in infertile women," Human Reproduction, vol. 11, no. 4, pp. 844849, 1996.

[23] X. B. Hua, C. R. Li, J. L. Zhou, D. N. Song, and Y. $\mathrm{L}$. Hu, "The development of atlas of acupuncture in rat," Laboratory Animal and Animal Experiment, vol. 1, pp. 1-5, 1991 (Chinese).

[24] V. Senna-Fernandes, D. L. França, D. de Souza, et al., “Acupuncture at 'Zusanli' (St.36) and 'Sanyinjiao' (SP.6) points on the gastrointestinal tract: a study of the bioavailability of $99 \mathrm{mTc}$-sodium pertechnetate in rats," Evidence-Based Complementary and Alternative Medicine. In press.

[25] N. Lédée-Bataille, G. Laprée-Delage, J.-L. Taupin, S. Dubanchet, R. Frydman, and G. Chaouat, "Concentration of leukaemia inhibitory factor (LIF) in uterine flushing fluid is highly predictive of embryo implantation," Human Reproduction, vol. 17, no. 1, pp. 213-218, 2002.

[26] H. Achache and A. Revel, "Endometrial receptivity markers, the journey to successful embryo implantation," Human Reproduction Update, vol. 12, no. 6, pp. 731-746, 2006.

[27] A. Makker and M. M. Singh, "Endometrial receptivity: clinical assessment in relation to fertility, infertility, and antifertility," Medicinal Research Reviews, vol. 26, no. 6, pp. 699-746, 2006.

[28] W. Tassell, M. Slater, J. A. Barden, and C. R. Murphy, "Endometrial cell death during early pregnancy in the rat," Histochemical Journal, vol. 32, no. 6, pp. 373-379, 2000.

[29] E. L. Parr, H. N. Tung, and M. B. Parr, "Apoptosis as the mode of uterine epithelial cell death during embryo implantation in mice and rats," Biology of Reproduction, vol. 36, no. 1, pp. 211225, 1987.

[30] J. Ordi, M. Creus, B. Ferrer et al., "Midluteal endometrial biopsy and $\alpha v \beta 3$ integrin expression in the evaluation of the endometrium in infertility: implications for fecundity," International Journal of Gynecological Pathology, vol. 21, no. 3, pp. 231-238, 2002.

[31] M. J. Murray, W. R. Meyer, R. J. Zaino et al., "A critical analysis of the accuracy, reproducibility, and clinical utility of histologic endometrial dating in fertile women," Fertility and Sterility, vol. 81, no. 5, pp. 1333-1343, 2004.

[32] J. Pei, E. Strehler, U. Noss et al., "Quantitative evaluation of spermatozoa ultrastructure after acupuncture treatment for idiopathic male infertility," Fertility and Sterility, vol. 84, no. 1, pp. 141-147, 2005.

[33] C.-1. Jin, K. Tohya, K. Kuribayashi, M. Kimura, and Y.H. Hirao, "Increased oocyte production after acupuncture treatment during superovulation process in mice," Journal of Reproduction and Contraception, vol. 20, no. 1, pp. 35-44, 2009.

[34] W. Yu, B. Horn, B. Acacio, D. Ni, R. Quintero, and M. Nouriani, "A pilot study evaluating the combination of acupuncture with sildenafil on endometrial thickness," Fertility and Sterility, vol. 87, pp. 439-442, 2007. 
[35] S. K. Dey, H. Lim, S. K. Das et al., "Molecular cues to implantation," Endocrine Reviews, vol. 25, no. 3, pp. 341-373, 2004.

[36] F. Liu, J. Xiong, G. Y. Huang, and W. Wang, "Study on the underlying mechanism of acupuncture in regulating neuroendocrine activity in dysmenorrhea rats," Zhen Ci Yan Jiu, vol. 34, no. 1, pp. 3-8, 2009 (Chinese).

[37] J. Zhou, F. Qu, X. Sang, X. Wang, and R. Nan, "Acupuncture and auricular acupressure in relieving menopausal hot flashes of bilaterally ovariectomized Chinese women: a randomized controlled trial," Evidence-Based Complementary and Alternative Medicine. In press.

[38] R. P. Dickey and D. E. Holtkamp, "Development, pharmacology and clinical experience with clomiphene citrate," Human Reproduction Update, vol. 2, no. 6, pp. 483-506, 1996.

[39] C. L. Cook, J. A. Schroeder, M. A. Yussman, and J. S. Sanfilippo, "Induction of luteal phase defect with clomiphene citrate," American Journal of Obstetrics and Gynecology, vol. 149, no. 6, pp. 613-616, 1984.

[40] J. Balasch, J. A. Vanrell, M. Duran, and J. GonzalezMerlo, "Luteal phase evaluation after clomiphene-chorionic gonadotrophin-induced ovulation," International Journal of Fertility, vol. 28, no. 2, pp. 104-106, 1983.

[41] C. L. Stewart, P. Kaspar, L. J. Brunet et al., "Blastocyst implantation depends on maternal expression of leukaemia inhibitory factor," Nature, vol. 359, no. 6390, pp. 76-79, 1992.

[42] M. J. Illera, P. L. Lorenzo, Y.-T. Gui, S. A. Beyler, K. B. C. Apparao, and B. A. Lessey, "A role for $\alpha \mathrm{v} \beta 3$ integrin during implantation in the rabbit model," Biology of Reproduction, vol. 68, no. 3, pp. 766-771, 2003.

[43] L. C. Kao, S. Tulac, S. Lobo et al., "Global gene profiling in human endometrium during the window of implantation," Endocrinology, vol. 143, no. 6, pp. 2119-2138, 2002.

[44] J. M. Borthwick, D. S. Charnock-Jones, B. D. Tom et al., "Determination of the transcript profile of human endometrium," Molecular Human Reproduction, vol. 9, no. 1, pp. 19-33, 2003.

[45] A. Riesewijk, J. Martín, R. van Os et al., "Gene expression profiling of human endometrial receptivity on days $\mathrm{LH}+2$ versus LH+7 by microarray technology," Molecular Human Reproduction, vol. 9, no. 5-6, pp. 253-264, 2003.

[46] Y.-S. Son, H.-J. Park, O.-B. Kwon, S.-C. Jung, H.-C. Shin, and S. Lim, "Antipyretic effects of acupuncture on the lipopolysaccharide-induced fever and expression of interleukin- 6 and interleukin- $1 \beta$ mRNAs in the hypothalamus of rats," Neuroscience Letters, vol. 319, no. 1, pp. 45-48, 2002.

[47] X.-Y. Liu, H.-F. Zhou, Y.-L. Pan et al., "Electroacupuncture stimulation protects dopaminergic neurons from inflammation-mediated damage in medial forebrain bundle-transected rats," Experimental Neurology, vol. 189, no. 1, pp. 189-196, 2004.

[48] F. J. Zijlstra, I. van den Berg-de Lange, F. J. P. M. Huygen, and J. Klein, "Anti-inflammatory actions of acupuncture," Mediators of Inflammation, vol. 12, no. 2, pp. 59-69, 2003.

[49] E. Stener-Victorin, U. Waldenström, M. Wikland, L. Nilsson, L. Hägglund, and T. Lundeberg, "Electro-acupuncture as a peroperative analgesic method and its effects on implantation rate and neuropeptide Y concentrations in follicular fluid," Human Reproduction, vol. 18, no. 7, pp. 1454-1460, 2003.

[50] J.-S. Kim, K. H. Shin, and C. S. Na, "Effect of acupuncture treatment on uterine motility and cyclooxygenase-2 expression in pregnant rats," Gynecologic and Obstetric Investigation, vol. 50 , no. 4 , pp. $225-230,2000$.
[51] H. L. Wang, L. Hu, and X. Z. Gao, "The effects of Sanyinjiao (SP-6) and Guanyuan (CV4) in increasing the gonadal hormone levels of menopausal model rats," Zhen Ci Yan Jiu, vol. 28, pp. 124-127, 2003 (Chinese).

[52] W. Y. Zhang, G. Y. Huang, and J. Liu, "Influences of acupuncture on infertility of rats with polycystic ovadian syndrome," Zhongguo Zhong Xi Yi Jie He Za Zhi, vol. 29, pp. 997-1000, 2009 (Chinese).

[53] X. Y. Liu, G. Y. Huang, and M. M. Zhang, "Preliminary study on the mechanisms of acupuncture in promoting embryo implantation in rats," Zhongguo Zhong Xi Yi Jie He Za Zhi, vol. 27, no. 7, pp. 633-636, 2007 (Chinese).

[54] C. Smith, M. Coyle, and R. J. Norman, "Influence of acupuncture stimulation on pregnancy rates for women undergoing embryo transfer," Fertility and Sterility, vol. 85, no. 5, pp. 1352-1358, 2006.

[55] L. G. Westergaard, Q. Mao, M. Krogslund, S. Sandrini, S. Lenz, and J. Grinsted, "Acupuncture on the day of embryo transfer significantly improves the reproductive outcome in infertile women: a prospective, randomized trial," Fertility and Sterility, vol. 85, no. 5, pp. 1341-1346, 2006.

[56] M. T. Lin, A. Chandra, S. M. Chen-Yen, and Y. F. Chern, "Needle stimulation of acupuncture loci Chu-Chih (LI-11) and $\mathrm{Ho}-\mathrm{Ku}$ (LI-4) induces hypothermia effects and analgesia in normal adults," American Journal of Chinese Medicine, vol. 9, no. 1, pp. 74-83, 1981.

[57] Q. C. Wang, Science of Acumox Therapeutics, China Press of Traditional Chinese Medicine, Beijing, China, 2003.

[58] H. Zhang, Z. Bian, and Z. Lin, "Are acupoints specific for diseases? A systematic review of the randomized controlled trials with sham acupuncture controls," Chinese Medicine, vol. 5, article 1, 2010.

[59] H. H. Moffet, "Sham acupuncture may be as efficacious as true acupuncture: a systematic review of clinical trials," Journal of Alternative and Complementary Medicine, vol. 15, no. 3, pp. 213-216, 2009.

[60] E. A. Macklin, P. M. Wayne, L. A. Kalish et al., "Stop Hypertension with the Acupuncture Research Program (SHARP): results of a randomized, controlled clinical trial," Hypertension, vol. 48, no. 5, pp. 838-845, 2006.

[61] L. A. Kalish, B. Buczynski, P. Connell et al., "Stop Hypertension with the Acupuncture Research Program (SHARP): clinical trial design and screening results," Controlled Clinical Trials, vol. 25, no. 1, pp. 76-103, 2004.

[62] H. M. Langevin and J. A. Yandow, "Relationship of acupuncture points and meridians to connective tissue planes," Anatomical Record, vol. 269, no. 6, pp. 257-265, 2002.

[63] A. C. Ahn, J. Wu, G. J. Badger, R. Hammerschlag, and H. M. Langevin, "Electrical impedance along connective tissue planes associated with acupuncture meridians," BMC Complementary and Alternative Medicine, vol. 5, article 10, 2005.

[64] C. M. Cassidy, "Moffet on the similarity of response to "active" and "sham" acupuncture," Journal of Alternative and Complementary Medicine, vol. 15, no. 3, pp. 209-210, 2009. 


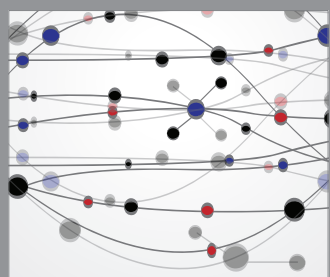

The Scientific World Journal
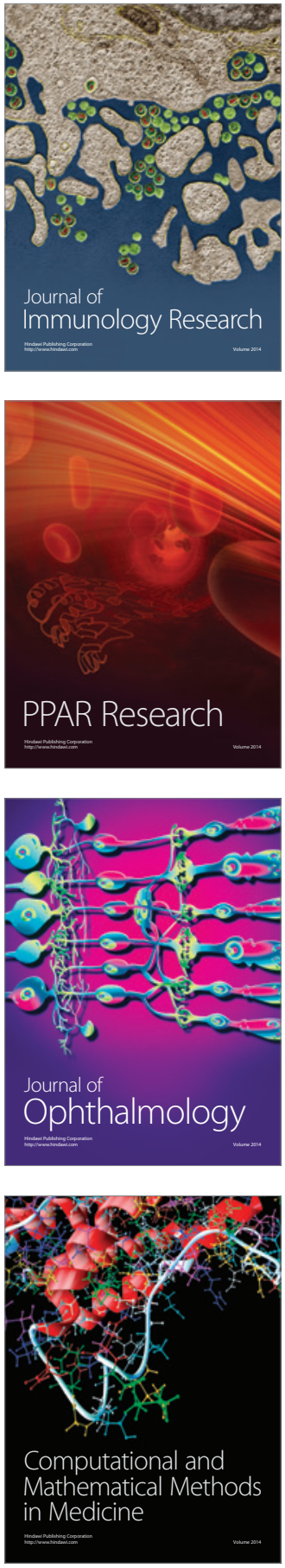

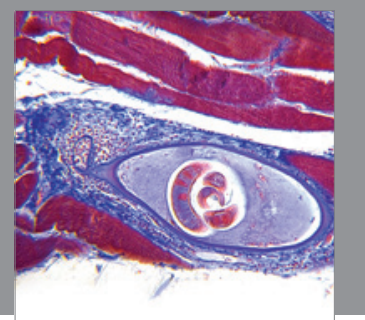

Gastroenterology

Research and Practice
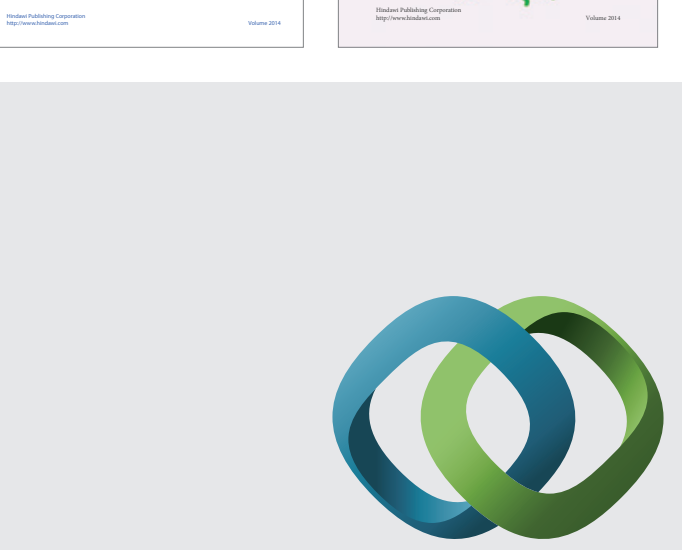

\section{Hindawi}

Submit your manuscripts at

http://www.hindawi.com
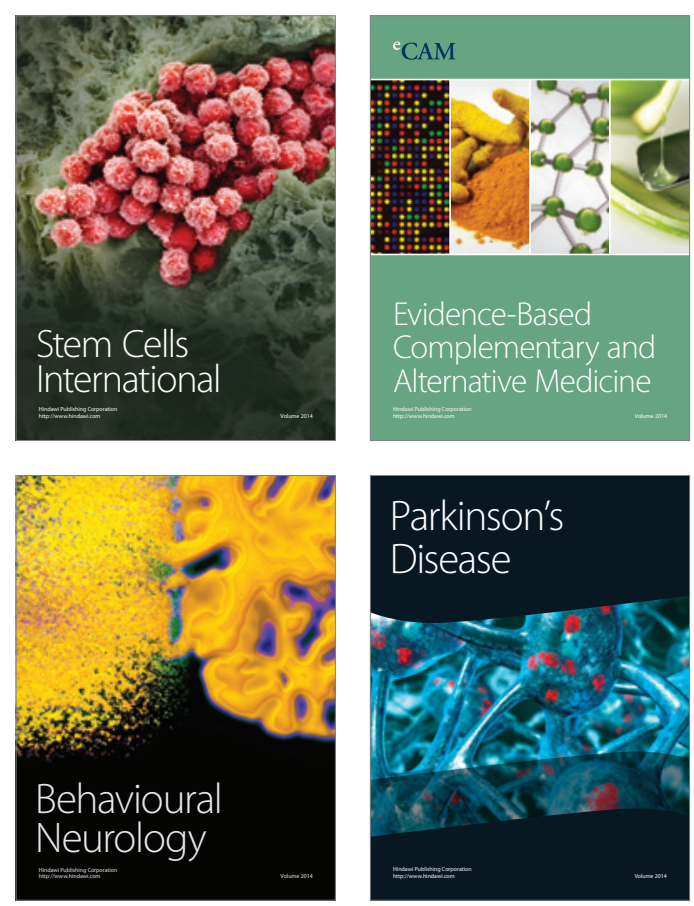

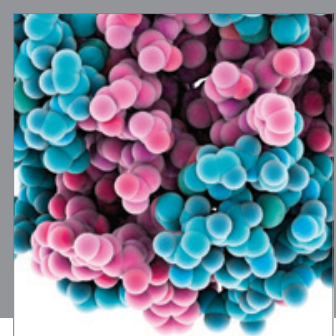

Journal of
Diabetes Research

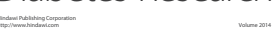

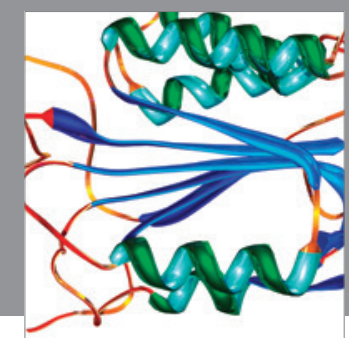

Disease Markers
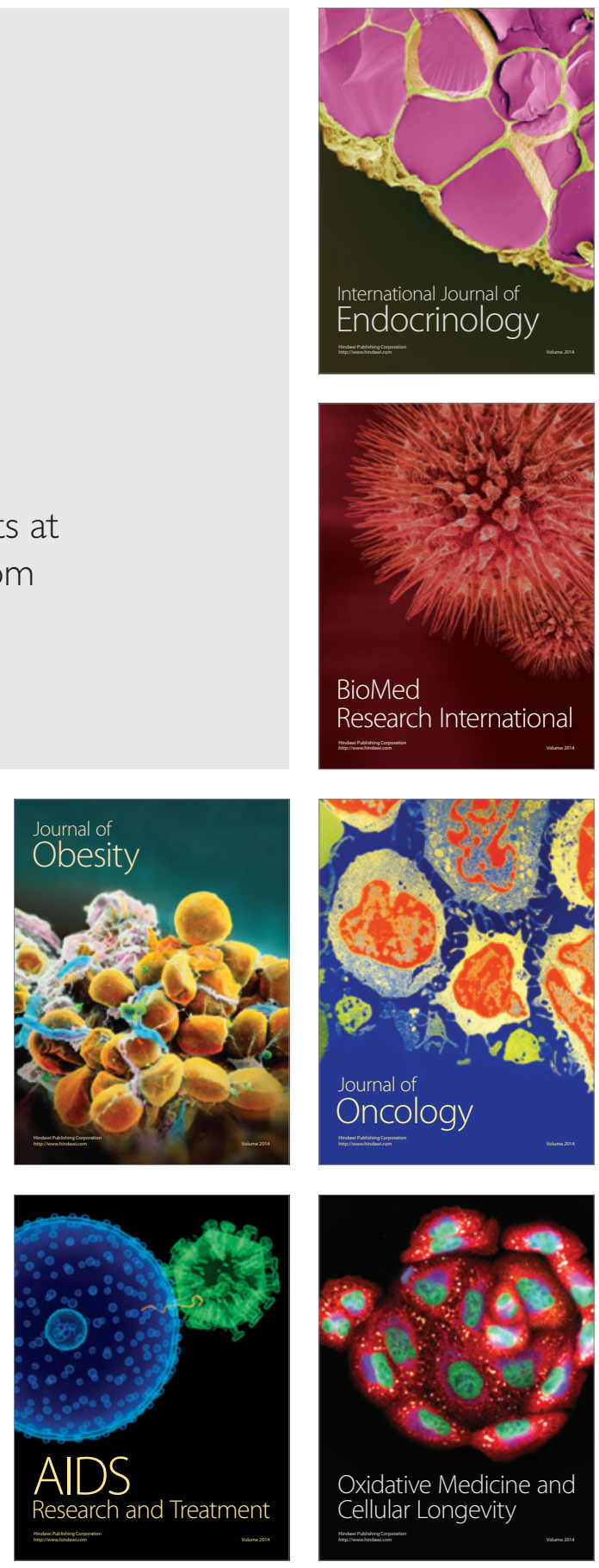